Original Research Paper

\title{
An Heuristic Approach Toward Innovative Tire Re-Design Through Advanced Technologies
}

\author{
Ramundo Fulvio Enzo and Fedele Lorenzo \\ Department of Mechanical and Aerospace Engineering, University "La Sapienza”, Rome, Italy
}

\author{
Article history \\ Received: 09-09-2019 \\ Revised: 18-07-2020 \\ Accepted: 17-08-2020 \\ Corresponding Author: \\ Ramundo Fulvio Enzo \\ Department of Mechanical and \\ Aerospace Engineering, \\ University "La Sapienza", \\ Rome, Italy \\ Email: fulvio.ramundo@uniroma1.it
}

\begin{abstract}
In the field of mobility and road transport, tires are components of a vehicle that play a decisive role in active safety. Monitoring tire conditions can affect different areas: Transport safety, optimization of cycles and maintenance costs and environmental protection. In fact, the tire wear check, possibly through the evaluation of the Remainig Useful Life (RUL), allows to meet many objectives: To ensure the necessary replacement of worn tires (safety target), to optimize the tire replacement cycles (maintenance target), avoid unnecessary early replacements (cost reduction target), reduce the amount of waste to be disposed of (environmental protection target). In this study two innovative methodologies, referable to technologies of Industry 4.0 field, are examined for the monitoring of tire wear: The first provides for direct monitoring through the use of "IoT" technology, the second provides for indirect monitoring through the use of "Big Data" technology. The scope and the limits of application are defined preliminarily, then the two methodologies are described according to the operating principle, in order to highlight merits and defects, finally a comparison of the two methodologies is provided. The proposed methodologies also allow to redesign the tires, developing new and safer products, with a flexible and integrated vision, which concerns the economic, social and environmental aspects from the perspective of managing the entire product life cycle (LCM - Life Cycle Management).
\end{abstract}

Keywords: Sustainable Tire Re-Design, Safety 4.0, Maintenance 4.0

\section{Introduction}

The condition of the tires is so important for the safety of road transport vehicles, that the state of wear cannot be left to the users of the vehicles: If we consider that the experimentation on autonomous driving of vehicles is constantly developing, it is not wrong to believe that vehicle users will devote themselves less and less to the direct control of the "state of health" of the vehicles.

Several functions are implemented directly on vehicles, which warn users, through messages on the display of the instrument panel, on the need to perform routine maintenance activities, no longer generally scheduled, but adaptive to the use of the vehicle itself.

Some functions are even able to prevent the use of the vehicle if not properly maintained. For example the need to periodically fill the AdBlue liquid tank, for diesel engines, whose complete exhaustion makes it impossible to use the vehicle itself until the conditions standards are restored: For this purpose the user is informed, progressively over time, of this necessity. There are already several sensors and functions, on the vehicles currently on the market, used to monitor the status of various components: For example tire pressure sensors are already widely used, while sensors and/or functions capable of monitoring tire wear status exist only at the level of patents (Sims, 1970; Thomas, 2007) or experimental studies (Matsuzaki and Todoroki, 2008; Longoria et al., 2019).

The automotive industry develops sensors and employs components to monitor the vehicle dynamics to ensure safety and comfort, consequently revealing a growing interest in intelligent tires; also the state of the art of scientific literature, related to tire monitoring, is constantly evolving as can be seen from the research and applications proposed in (Hariri et al., 2017; Coppo et al., 2017; Egaji et al., 2019): Industrial and academic research is directed mainly towards the development of technologically advanced sensors, thus moving towards the development of IoT applications.

On the other hand, this research aims to achieve similar goals using "Big Data" technology: More 
precisely and with regard to tire wear monitoring, this article shows, proposes and compares innovative approaches, which use "Big Data" or "IoT" technologies, spreading widely and rapidly thanks to the recent context of Industry 4.0.

Through the proposed methodologies it is possible to rethink and redesign the tires, developing new and safer products, with the pursuit of economic, social and environmental sustainability: By systematically integrating the concepts of sustainability, eco-design and the application of the Best Available Technologies (BAT) it is possible to pursue sustainable production and consumption, optimization of the production process in technological-structural terms, exploitation of resources, downstream impact on the ecosystem and energy consumption, managing the entire product life cycle (LCM - Life Cycle Management).

The rest of the document is organized as follows: Section 2 introduces the "IoT" and "Big Data" technology; section 3 describes the methodologies proposed for implementing the 4.0 technologies in tire monitoring; section 4 shows a comparative study among the proposed methodologies highlighting their strengths and weaknesses; section 5 contains the authors' conclusions on the article.

\section{Technologies}

\section{IoT - Internet of Things}

Ashton (2009) co-founder of the Auto-ID Center at MIT, first mentioned the internet of things in 1999 linking the new idea of Radio Frequency Identification (RFID) in supply chain to the hot topic of the Internet; it is worth remembering that MIT Professor Neil Gershenfeld in his book, When Things Start to Think also appearing in 1999, didn't use the exact term but provided a clear vision of where IoT was headed.

For an extensive study on the IoT concept it is appropriate to refer to a specific and detailed survey (Atzori et al., 2010), while in this study we will report only some basic steps in the evolution of IoT technology.

The internet of things, or IoT, is a system of interrelated computing devices, mechanical and digital machines, objects or people that are provided with Unique Identifiers (UIds) and the ability to transfer data over a network without requiring human-to-human or human-to-computer interaction.

A thing, in the internet of things, is any natural or manmade object that can be assigned an IP address and is able to transfer data over a network. IoT technology evolved thanks to the convergence of wireless technologies, Microelectromechanical Systems (MEMS), microservices and the Internet, breaking up the separation between Operative Technology (OT) and Information Technology (IT) and allowing to analyze the unstructured data generated from machines to obtain useful information to improve performance.
IoT also evolved from communication between machines that connect to one another through a network without human interaction (Machine-to-Machine-M2M): By bringing $\mathrm{M} 2 \mathrm{M}$ to the next level, IoT is a sensor network of numerous smart devices that connect people, systems and other applications to collect and share data.

An IoT system consists of web-enabled smart devices that use embedded processors, sensors and communication hardware to collect, send and act on data they acquire from their environments. IoT devices share the sensor data they collect by connecting to an IoT gateway or other edge device where data is either sent to be analyzed. Sometimes, these devices communicate with other related devices and act on the information they get from one another. The devices do most of the work without human intervention, although people can interact with the devices for instance, to set them up, give them instructions or access the data.

The connectivity, networking and communication protocols used with these web-enabled devices largely depend on the specific IoT applications deployed.

\section{Tire Monitoring Methodologies}

\section{Big Data}

The The term "Big Data" has been in use since the 1990s: It is believed in the literature that (John, 1998) popularized the term (Lohr, 2013). Big data philosophy encompasses unstructured, semi-structured and structured data: Big data requires a set of techniques and technologies with new forms of integration to reveal insights from datasets that are diverse, complex and of a massive scale (Sriramoju, 2017). Therefore Big data is an evolving term that describes a large volume of data that has the potential to be mined for information and used in machine learning projects and other advanced analytic applications.

The existing definitions for Big Data provide very different perspectives: Big Data is considered in turn as a term describing a social phenomenon, information assets, data sets, storage technologies, analytical techniques, processes and infrastructures (De Mauro et al., 2016). A 2016 definition states that "Big data represents the information assets characterized by such a high volume, velocity and variety to require specific technology and analytical methods for its transformation into value" (De Mauro et al., 2016).

Big Data uses inductive statistics and concepts from nonlinear system identification to infer laws (regressions, nonlinear relationships and causal effects) from large sets of data with low information density to reveal relationships and dependencies, or to perform predictions of outcomes and behaviors (Mishra et al., 2016).

Big Data is often characterized by the $3 \mathrm{Vs}$ that were first identified by Gartner analyst, (Laney, 2001) in a report published in: The extreme volume of data, the 
wide variety of data types and the velocity at which the data must be processed.

Ultimately, the value and effectiveness of Big Data depends on the workers tasked with understanding the data and formulating the proper queries to direct Big Data analytics projects: Some Big Data tools meet specialized niches and allow technical users to use everyday data in predictive analytics applications.

\section{Tire Monitoring by IoT}

The use of IoT technology provides that objects can send data on their operating and/or health conditions: In this case the tires must be able to send data, on their wear and tear, to an on-board computer of the vehicle in order to communicate the useful information to the driver and possibly to a database of the tire manufacturer for the development of new and safer products.

Internet of Things (IoT) can play a key role in reducing the uncertainty related to the conditions and remaining lives of the returned EOL products, with the help of sensors and RFID tags, which then can be used to decide a feasible recovery process for the EOL product amongst disassembly, remanufacturing, recycling or disposal (Joshi and Guptab, 2019).

Currently there are no tires with integrated technologies able to transfer data on the market; however, there are sensors able to do it in their place: Think, for example, of the pressure sensors already widespread in the vehicles marketed. Likewise, it can be considered plausible to develop a sensor capable of detecting the thickness of the tread of a tire.

Therefore the methodology, in tire monitoring, through the use of IoT technology is really essential, since it refers directly to the technology devised and developed for the operation of a specific sensor able to detect the thickness of a tire and to the possibility of transferring this information in radio frequency mode.

This technology, even if not yet commercialized, is already available: A fully printed sensor, made of metallic carbon nanotube ink, capable of noninvasive material thickness detection was already developed and tested (Andrews et al., 2017). By applying the sensors directly beneath the tread (within the tire), changes in the tread depth are able to be detected: This is a low-cost approach for monitoring changes in material thickness, using noninvasive printed sensors, applicable in monitoring tire wear by Internet-of- Things (IoT) technology.

The following Fig. 1 shows the positioning of the sensor inside the tire.

In the article (Andrews et al., 2017) the authors declare that "this proof- of-concept experiment provides reinforcement to the applicability of these noninvasive material thickness sensors and demonstrates a different measurement technique to allow sensing despite the steel mesh in a car tire", but no detailed results are provided on the reliability of this tire monitoring methodology during their life cycle.

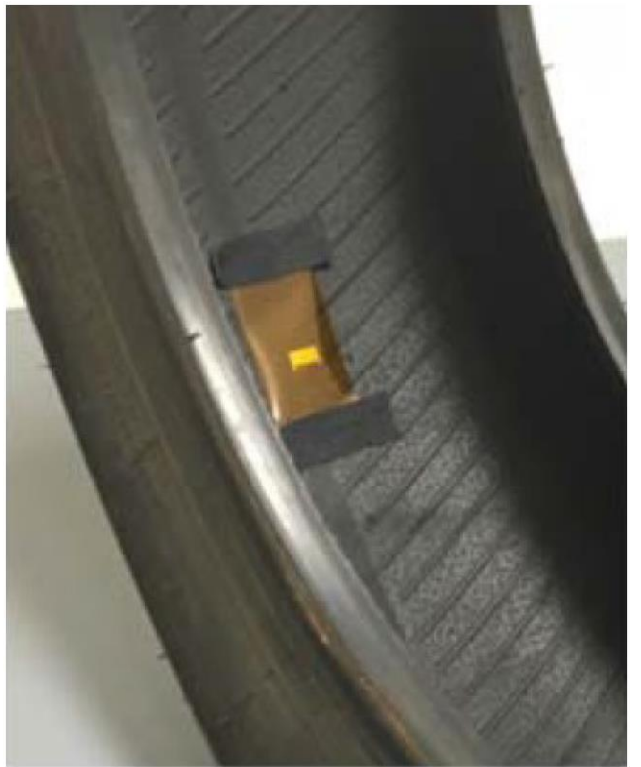

Fig. 1: A photograph of the IoT metalliccarbon nanotube ink sensor

Therefore, it is a method of direct measurement of tire wear, whose strengths are:

- Accurate detection of the tread thickness by direct measurement

- Monitoring the wear of the tread of each tire

- Possibility of preparing an RFID to monitor the entire life cycle of the tire

- Low cost of sensors

- Independent of tire pressure

- Not necessary to install a GPS system on the vehicle

- While the weaknesses are

- Need to adapt the vehicle to receive data from the sensors

- $\quad$ Risk that the sensor may break, for example due to impact during the tire rolling and become ineffective

\section{Tire Monitoring by Big Data}

The use of Big Data technology instead provides the development of a more detailed methodology, in fact the state of wear of a tire does not occur through a direct measurement of the tread thickness, but through the interpretation of data attributable to it: It is therefore of an indirect type of monitoring.

The idea of performing a remote control originates from the concept of "Tele-Maintenance", already expressed in (Concetti et al., 2009) and in (Fedele, 2011) with the aim of efficiently achieving the goal of optimizing the maintenance activities, in terms of quality and cost, through an "intelligent" maintenance management system for remote monitoring and control of plants and machines.

In this article, starting from the idea of remote control, we propose a methodology that provides for the analysis of 
the variability of the data referred to the vehicle's GPS speed, variability caused by the progressive consumption of the tires, which emerges from the comparison with the velocity indicated by the vehicle speedometer.

As is known, the speed displayed by the speedometer of a vehicle is always greater, or more equal, to the true speed of the vehicle UN-ECE, 2004, in fact the following relation is valid:

$$
0 \leq V d-V t \leq 0,1 * V t+4
$$

Where:

$V d=$ Speed displayed by the tachometer espressed in [Km/h]

$V t=$ The true speed of the veicle espressed in $[\mathrm{Km} / \mathrm{h}]$

We assume that true speed $V t$ is equal to the speed detected by GPS system, in consideration of the high accuracy of the GPS tachometers available today:

$$
0 \leq V d-V g p s \leq 0,1 * V g p s+4
$$

The speed $V d$, displayed by the vehicle speedometer is usually measured according to the number of revolutions of the transmission shaft and is therefore insensitive to the consumption of the tread: Therefore the tachometer will indicate the same speed values in the presence of new and worn tires.

While at the same speed $V d$, displayed by the tachometer, different values will be obtained for the VgpsN speed, measured with new tread and the VgpsU speed, measured with worn out tread, precisely as a consequence of the reduction of the tire's circumference. Furthermore, for the study treated, a widespread type of tire is taken into consideration, the characteristics of which are reported in Table 1.

A new tread thickness of $8 \mathrm{~mm}$ and a worn tread thickness of $1.6[\mathrm{~mm}]$ is also considered.

The methodology therefore envisages a first learning phase, carried out in the first 2,000 [km] of the new tire and during which the $\mathrm{VgpsN}$ and $\mathrm{Vd}$ velocity data are associated.

In Table 2 is shown the correlation between speeds: The values are expressed in $[\mathrm{Km} / \mathrm{h}]$ and VgpsN is the speed detected, through GPS, in conditions of tire with new tread.

As the kilometers traveled by the tires increase, or as a consequence of the wear of the tread, the values of Vgps, compared with those of Vd, will show a decrease: It is assumed to detect the speed data, when the tread thickness reaches the limit value of 1.6 [mm].

Table 3 shows the correlations between Vd, VgpsN and $\mathrm{VgpsU}$, espressed in $[\mathrm{Km} / \mathrm{h}]$. VgpsU is the speed detected, using a GPS system, in the presence of a worn tire with a tread limit thickness of 1.6 [mm].

It is therefore possible to calculate the variations of the quantities, as shown in Table 4, in which.
Table 1: Characteristic dimensions of tyre

\begin{tabular}{ll}
\hline Tire Size & $205 / 55 \mathrm{R} 16$ \\
\hline Section width [mm] & 205 \\
Tire shape ratio & 55 \\
Rim diameter [mm] & 406,4 \\
Theoric sidewall height [mm] & 112,8 \\
Theoric overall diameter [mm] & 631,9 \\
Theoric circumference [mm] & 1985,2 \\
\hline
\end{tabular}

Table 2: Correlation between VgpsN and Vd

\begin{tabular}{ll}
\hline VgpsN & Vd \\
\hline 0 & 0 \\
10 & 10,7 \\
20 & 21,4 \\
30 & 32,1 \\
40 & 42,8 \\
50 & 53,5 \\
60 & 64,2 \\
70 & 74,9 \\
80 & 85,6 \\
90 & 96,3 \\
100 & 107 \\
110 & 117,7 \\
120 & 128,4 \\
130 & 139,1 \\
\hline
\end{tabular}

Table 3: Correlations between Vd, VgpsN and VgpsU

\begin{tabular}{lcl}
\hline $\mathrm{Vd}$ & VgpsN & VgpsU \\
\hline 0 & 0 & 0 \\
10,7 & 10 & 9,8 \\
21,4 & 20 & 19,6 \\
32,1 & 30 & 29,4 \\
42,8 & 40 & 39,2 \\
53,5 & 50 & 49 \\
64,2 & 60 & 58,8 \\
74,9 & 70 & 68,6 \\
85,6 & 80 & 78,4 \\
96,3 & 90 & 88,2 \\
107 & 100 & 98 \\
117,7 & 110 & 107,8 \\
128,4 & 120 & 117,6 \\
139,1 & 130 & 127,4 \\
\hline
\end{tabular}

Table 4: Speed differentials

\begin{tabular}{llll}
\hline Vgps & Vd-VgpsN & Vd-VgpsU & $\Delta$. Vgps \\
\hline 0 & 0 & 0 & 0 \\
10 & 0,7 & 0,9 & 0,2 \\
20 & 1,4 & 1,8 & 0,4 \\
30 & 2,1 & 2,7 & 0,6 \\
40 & 2,8 & 3,6 & 0,8 \\
50 & 3,5 & 4,5 & 1 \\
60 & 4,2 & 5,4 & 1,2 \\
70 & 4,9 & 6,3 & 1,4 \\
80 & 5,6 & 7,2 & 1,6 \\
90 & 6,3 & 8,1 & 1,8 \\
100 & 7 & 9 & 2 \\
110 & 7,7 & 9,9 & 2,2 \\
120 & 8,4 & 10,8 & 2,4 \\
130 & 9,1 & 11,7 & 2,6 \\
\hline
\end{tabular}


It is therefore possible to calculate the variations of the quantities, as shown in table 4, in which:

$(\mathrm{Vd}-\mathrm{VgpsN})=$ The difference between $\mathrm{Vd}$ and $\mathrm{VgpsN}$ $(\mathrm{Vd}-\mathrm{VgpsU})=$ The difference between $\mathrm{Vd}$ and $\mathrm{VgpsU}$ $\Delta$.Vgps $=$ The difference between $\mathrm{VgpsN}$ and VgpsU

The trend of speed variations, caused by tire wear, is shown in Fig. 2.

Comparing the values of $\Delta$.Vgps and the values (Vd$\mathrm{VgpsN}$ ) a maximum percentage variation of $28.57 \%$ is detected, between a new tire with a tread depth of $8 \mathrm{~mm}$ and a worn tire with a tread depth of $1.6 \mathrm{~mm}$. It is therefore possible to define an indicator on the tire wear status as follows:

$$
T H I=\Delta . V g p s \times 100 /(V d-V g p s N)
$$

in which THI means Tire Health Indicator.

Based on the results, it is considered appropriate to establish a maximum value of the THI equal to $28 \%$, above which the tires, of the type taken into consideration, must be replaced.

It is also possible to define suitable intervals, as the THI value varies, to provide useful information to the driver of a vehicle, such as the indication on consumption and the Residual Useful Life (RUL) of the tires: An example to derive RUL from THI parameter is shown in Table 5.

$\square \mathrm{Vd}-\mathrm{VgpsN} \diamond \mathrm{Vd}-\mathrm{Vgps} \mathrm{V} \quad \Delta$.Vgps

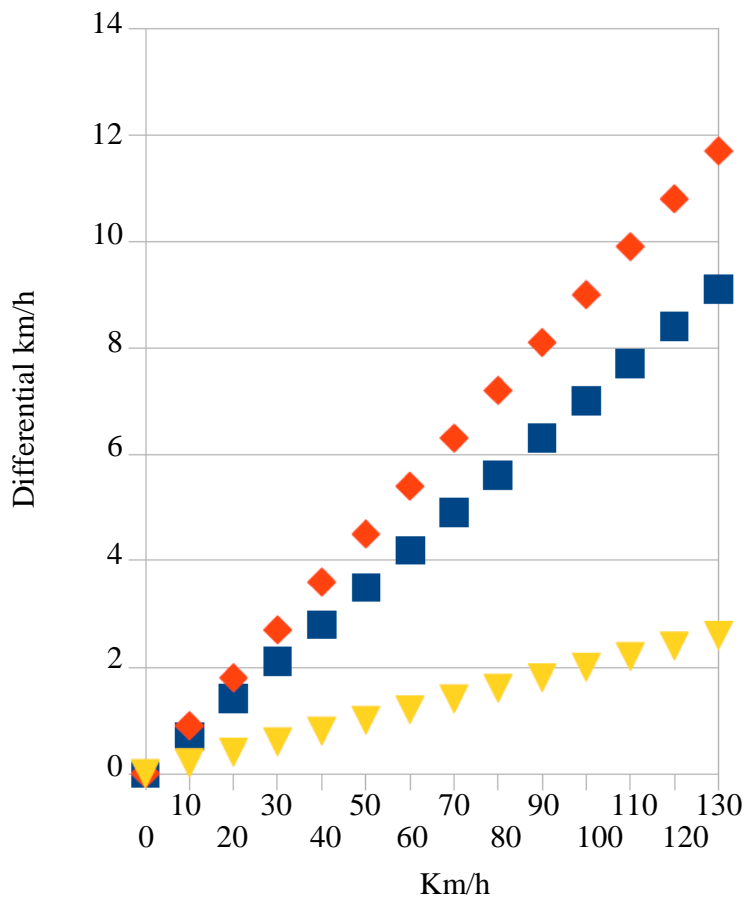

Fig. 2: Trend of speed variations
The methodology proposed is an indirect measurement of tire wear, whose strengths are:

- Useful tread thickness detection

- Monitoring tire tread wear

- Possibility to detect the tread thickness starting from the speed data

- Low cost of development and implementation

- Independent of tire pressure

- Not necessary to install sensors inside the tires; while the weaknesses are

- Necessary to install a GPS system on the vehicle

- Not possible monitoring the wear of the tread of each tire

\section{Comparison of 4.0 Technologies in Tire Wear Monitoring}

The main difference between the methodologies described derives from the relative technology used: detection of the tread thickness with direct measurement by IoT and with indirect measurement by Big Data.

The following Table 6 shows a comparative analysis between the two methodologies envisaged.

Table 5: Correlation between THI and RUL

\begin{tabular}{lcc}
\hline THI & \% Tire consumption & \%RUL \\
\hline 7 & 25 & 75 \\
14 & 50 & 50 \\
21 & 75 & 25 \\
28 & 100 & 0 \\
\hline
\end{tabular}

Table 6: Comparative analysis between IoT and Big Data methodologies

\begin{tabular}{|c|c|c|}
\hline Comparison features & IoT & Big Data \\
\hline $\begin{array}{l}\text { Accurate detection of the tread } \\
\text { thickness by direct measurement }\end{array}$ & $\checkmark$ & \\
\hline $\begin{array}{l}\text { Useful detection of the tread } \\
\text { thickness by indirect measurement }\end{array}$ & & $\checkmark$ \\
\hline $\begin{array}{l}\text { Monitoring the wear of the tread } \\
\text { of each tire }\end{array}$ & $\checkmark$ & \\
\hline Monitoring tire tread wear & & $\checkmark$ \\
\hline $\begin{array}{l}\text { Possibility of preparing an RFID to } \\
\text { monitor the entire life cycle of the tire }\end{array}$ & & $\checkmark$ \\
\hline $\begin{array}{l}\text { Possibility to detect the tread } \\
\text { thickness starting from the speed data }\end{array}$ & $\checkmark$ & \\
\hline $\begin{array}{l}\text { Not necessary to install a GPS system } \\
\text { on the vehicle }\end{array}$ & $\checkmark$ & $\checkmark$ \\
\hline $\begin{array}{l}\text { Not necessary to install sensors } \\
\text { inside the tire }\end{array}$ & & \\
\hline $\begin{array}{l}\text { Low cost of sensors installed } \\
\text { inside the tire }\end{array}$ & $\begin{array}{l}\checkmark \\
\checkmark\end{array}$ & \\
\hline $\begin{array}{l}\text { Low cost of development and } \\
\text { implementation of methodology } \\
\text { of monitoring }\end{array}$ & & $\checkmark$ \\
\hline Independent of tire pressure & $\checkmark$ & $\checkmark$ \\
\hline
\end{tabular}




\section{Conclusion}

The study proposed in this study takes into consideration the possibility of monitoring tire wear on vehicles, comparing two different methodologies through the use of Innovative Technologies (IoT, Big Data) in order to achieve the following objectives:

- Ensure the necessary replacement of worn tires (safety target)

- Optimize the tire replacement cycles (maintenance target)

- Avoid unnecessary early replacements (cost reduction target)

- Reduce the amount of waste to be disposed of (environmental protection target)

The development, implementation and use of a tire wear monitoring system requires to investigate some technical, economic, social and ethical issues, since as previously observed, such a system offers the possibility of using the tires up to the tread wear limit indicated by the regulations $(1.6 \mathrm{~mm})$.

In a study conducted by a tire manufacturer (Website Michelin Road Usage Lab) it is stated that every year in Europe, if all the tires were used up to the legal wear limit, that is $1.6 \mathrm{~mm}$, they would be avoided:

- $\quad 128$ Million tyres would be saved

- $€ 6.9$ Billion Expenses saved for drivers

- $\quad$ 6.6 Million tons of $\mathrm{CO}_{2}$ saved

Also with regards to performance, it is widely believed that a tire with a partially worn tread has lost part of its efficiency: This conviction has no real confirmation in the experimental tests conducted by the same tire manufacturer that ensures similar performance for new and worn tires.

The adoption of a tire wear methodology, with IoT or Big Data technology, has consequences in terms of costs and benefits. The benefits obtainable can be summarized as follows:

- Improvement of active safety when driving vehicles

- Possibility of inhibiting the start and use of vehicles with excessively worn tires

- Possible reduction of accidents deriving from the circulation of vehicles with excessively worn tires

- Possibility of defining a maintenance plan, through the tire RUL

- Reduction of rubber materials to be disposed of and/or recycled

- Less use of raw materials to be used in the production of new tires
With regard to costs, the following consequences occur:

- Higher costs deriving from the development of tire wear monitoring methodologies

- Higher costs resulting from the implementation of tire wear monitoring technologies

- Lower costs resulting from a reduction in the number of accidents caused by vehicles circulating with tires that are Excessively worn lower costs resulting from early tire replacement

- Lower costs due to the disposal/recycling of rubber materials

- Lower costs for use reduction of raw materials to be used in the production of new tires

From a technical point of view the most important difference that emerges from the comparison of the two methodologies proposed, is in the method and accuracy of the tire tread thickness measurement: Direct and accurate through the IoT technology, indirect and useful through Big Data technology.

However, it is necessary to underline that the IoT technology requires the predisposition of specific sensors inside the tires, which in addition to having their own cost could be subject to malfunctions and/or breakages as a result of shock; on the other hand the Big Data technology need a GPS system installed on the vehicle, whose installation is already widely spread on the vehicles trade.

A more important limitation of the proposed methodology with Big Data technology is the inability to monitor the tread wear of a vehicle's single tire: Monitoring in fact, based on a continuous comparison between the vehicle's GPS speed and the speed displayed on the vehicle speedometer (generally detected by the revolutions of the transmission shaft), it is referable to the driving wheels of the vehicle.

Future perspectives of the study carried out consists precisely in overcoming this limit, i.e., developing a methodology, based on Big Data technology, which is able to monitor the tread wear of each tire of a vehicle.

\section{Author's Contributions}

Lorenzo Fedele: Coordinated the research activity, indicating the guidelines and providing feedback on the review activity.

Fulvio Ramundo: Led the development of the research and managed the activities necessary for publication.

\section{Ethics}

This article is original and contains unpublished material. The corresponding author confirms that all of the other authors have read and approved the manuscript and no ethical issues involved. 


\section{References}

Andrews, J. B., Cao, C., Brooke, M. A., \& Franklin, A. D. (2017). Noninvasive material thickness detection by aerosol jet printed sensors enhanced through metallic carbon nanotube ink. IEEE Sensors Journal, 17(14), 4612-4618.

Ashton, K. (2009). That 'internet of things' thing. RFID journal, 22(7), 97-114.

Atzori, L., Iera, A., \& Morabito, G. (2010). The internet of things: A survey. Computer networks, 54(15), 2787-2805.

Brey, T. A. (2007). U.S. Patent No. 7,180,409. Washington, DC: U.S. Patent and Trademark Office.

Concetti, M., Cuccioletta, R., Fedele, L., \& Mercuri, G. (2009). Tele-maintenance "intelligent" system for technical plants result management. Reliability Engineering \& System Safety, 94(1), 63-77.

Coppo, F., Pepe, G., Roveri, N., \& Carcaterra, A. (2017). A multisensing setup for the intelligent tire monitoring. Sensors, 17(3), 576.

De Mauro, A., Greco, M., \& Grimaldi, M. (2016). A formal definition of Big Data based on its essential features. Library Review.

Egaji, O. A., Chakhar, S., \& Brown, D. (2019). An innovative decision rule approach to tyre pressure monitoring. Expert systems with applications, 124, 252-270.

Fedele, L. (2011). Methodologies and techniques for advanced maintenance. Springer Science \& Business Media.

Hariri, H., Kim, J., Kim, W. S., Frechette, L. G., \& Masson, P. (2017). Performance validation of printed strain sensors for active control of intelligent tires. Applied Acoustics, 123, 73-84.

John, R. M. (1998). Big data and the next wave of infrastress. Slides From Invited Talk.
Joshi, A. D., \& Gupta, S. M. (2019). Evaluation of design alternatives of End-Of-Life products using internet of things. International Journal of Production Economics, 208, 281-293.

Laney, D. (2001). 3D data management: Controlling data volume, velocity and variety. Gartner. http://blogs.gartner.com/douglaney/files/2012/01/ad949-3D-DataManagementControlling-Data-Volume-Velocityand-Variety.pdf

Lohr, S. (2013). The origins of 'Big Data': An etymological detective story. New York Times, 1(1).

Longoria, R. G., Brushaber, R., \& Simms, A. (2019). An in-wheel sensor for monitoring tire-terrain interaction: Development and laboratory testing. Journal of Terramechanics, 82, 43-52.

Matsuzaki, R., \& Todoroki, A. (2008). Wireless monitoring of automobile tires for intelligent tires. Sensors, 8(12), 8123-8138.

Mishra, B. S. P., Dehuri, S., \& Kim, E. (Eds.). (2016). Techniques and environments for big data analysis: parallel, cloud and grid computing (Vol. 17). Springer.

Sims, C. D. (1970). U.S. Patent No. 3,516,467. Washington, DC: U.S. Patent and Trademark Office.

Sriramoju, S. B. (2017). Introduction to big data: infrastructure and networking considerations. Horizon Books (A Division of Ignited Minds Edutech P Ltd).

Website Michelin Road Usage Lab. www.michelin.co.uk/michelin-tyreperformances/long-lastingperformances\#environmental 